\title{
Plasmon-assisted Energy Transfer near Coated Metal Cylinders
}

\author{
Vasilios D. Karanikolas, Cristian A. Marocico and A. Louise Bradley \\ Semiconductor Photonics Group, School of Physics \\ Trinity College Dublin, College Green 2, Dublin, Ireland \\ e-mail:bradlel@tcd.ie
}

\begin{abstract}
In this contribution we consider the decay and energy transfer rates of dipole emitters near dielectric-coated metal cylinders. The surface plasmons excited at the surface of the metal core can act as a very efficient channel both for increasing the decay rate of the emitter or for the transfer of energy from one dipole to another.
\end{abstract}

Keywords: energy transfer, surface plasmons, decay rates, coated metal cylinder

\section{INTRODUCTION}

Starting with Purcell's early work on this topic [1], it has been known that the electromagnetic properties of quantum emitters (fluorescent molecules, quantum dots) are not intrinsic, but can be modified by its surroundings. The decay rate of a quantum emitter has been investigated in a number of different geometries, such as planar interfaces [2-4], spheres [5, 6] and nanowires [7,8]. Similarly, the rate of energy transfer between quantum systems has been studied intensively, both theoretically and experimentally in such systems as thin metal films $[4,9,10]$, nanowires [8], spheres [11, 12]. The process of resonant transfer of excitation energy between two quantum systems is the main mechanism by which an excited system transfers its electronic excitation to a near-neighbour. As such, it plays an essential role in microscopy, nanophotonics, biophysics, etc.

In this contribution we consider the decay and energy transfer rates of emitters near dielectrically coated metal cylinders. These cylinders support surface-plasmon polaritons (SPPs) and localized surface-plasmons, which are modes of the electromagnetic field characterized by strong confinement (sub-wavelength) to an interface and large values of the field intensity. Section 2 sets up the theoretical framework used in this investigation, namely that of the Green's tensor. Section 3 presents the results of numerical simulations performed within the Green's tensor formalism, as well as a discussion of these results. Finally, Section 4 is reserved for conclusions and outlook.

\section{THEORETICAL FRAMEWORK}

We now consider the theoretical framework used to model the decay and energy transfer rates in the presence of a coated metal cylinder. For this we use a Green's tensor technique, widely employed for this type of investigation and readily amenable to numerical work.

\subsection{Decay and energy transfer rates in the Green's tensor formalism}

The decay rate of an emitter placed in any surroundings can be calculated with the aid of the Green's tensor for the surroundings, as [10]

$$
\gamma(\mathbf{r}, \omega)=\frac{2 \mu^{2} \omega^{2}}{\hbar c^{2} \varepsilon_{0}} \operatorname{Im}[\hat{\boldsymbol{\mu}} \cdot \mathfrak{G}(\mathbf{r}, \mathbf{r}, \omega) \cdot \hat{\boldsymbol{\mu}}]
$$

where $\boldsymbol{\mu}$ is the transition dipole moment of the emitter between its excited and ground states, and G(r, r, $\omega)$ is the Green's tensor of the electromagnetic field at the position $\mathbf{r}$ of the emitter. Similarly, the energy transfer rate between a donor dipole located at position $\mathbf{r}_{\mathrm{D}}$ and an acceptor dipole located at position $\mathbf{r}_{\mathrm{A}}$ can be written in terms of the Green's tensor thus:

$$
\Gamma\left(\mathbf{r}_{A}, \mathbf{r}_{D}, \omega\right)=\frac{2 \pi}{\hbar^{2}}\left(\frac{\omega^{2}}{\varepsilon_{0} c^{2}}\right)^{2}\left|\boldsymbol{\mu}_{A} \cdot \mathfrak{G}\left(\mathbf{r}_{A}, \mathbf{r}_{D}, \omega\right) \cdot \boldsymbol{\mu}_{D}\right|^{2}
$$

with the Green's tensor taken now between the positions of the donor and acceptor. The expressions above have been derived with the assumption that the emission and absorption spectra of the donor and acceptor are very narrow compared to the frequency interval over which the Green's tensor varies appreciably. When considering particular cases of donors and acceptors, one must weigh the expressions above with their emission and absorption spectra to obtain the real rates. With this understanding, we shall use the expressions above in this contribution. Furthermore, we wish to compare the decay and energy transfer rates in the presence of the coated metal cylinder with those in free space. To this end, we shall normalize the rates given above with the rates in free space and present our results for these normalized rates. 


\subsection{The Green's tensor for a coated cylinder}

The approach we have taken here to investigate the decay and energy transfer rates is a semi-analytical approach and knowledge of the Green's tensor for the coated cylinder geometry is required. In order to obtain the Green's tensor for this geometry we begin with the Green's tensor of a homogeneous medium expanded in cylindrical vector wavefunctions. This expansion reads:

$$
\mathcal{G}\left(\mathbf{r}_{A}, \mathbf{r}_{D}, \omega\right)=\frac{i}{8 \pi} \sum_{n, L} \int \mathrm{d} k_{z} \frac{1}{k_{\rho}^{2}} \mathbf{H}_{L}\left(\mathbf{r}_{A}\right) \otimes \mathbf{J}_{L}\left(\mathbf{r}_{D}\right)
$$

where the sum is over the angular number $n$ and the polarization index $L$. The $\mathbf{H}$ and $\mathbf{J}$ are cylindrical vector wavefunctions containing the Hankel or Bessel functions $H$ or $J$, respectively. Finally, we have $k_{\rho}^{2}=k^{2}-k_{z}^{2}$ where $k$ is the wavevector and we integrate over the $z$-component of the wavevector, $k_{z}$.

From this form of the Green's tensor in a homogeneous medium, one can obtain the Green's tensor of a coated cylinder by expanding in a similar way the Green's tensor in each of the media comprising the geometry (core, coating, background) and imposing the usual continuity conditions at the interfaces between these media. Though somewhat involved analytically, this procedure does not present any conceptual difficulty and we will not present it here.

\section{RESULTS AND DISCUSSION}

We will present in this contribution simulation results for the decay rate of an emitter placed close to a coated metallic cylinder and the energy transfer rate between a donor and an acceptor in the vicinity of the same. A schematic of the coated metal cylinder with its geometric and material parameters is presented in Fig. 1. We begin with the decay rate of a quantum emitter near the coated metallic cylinder.

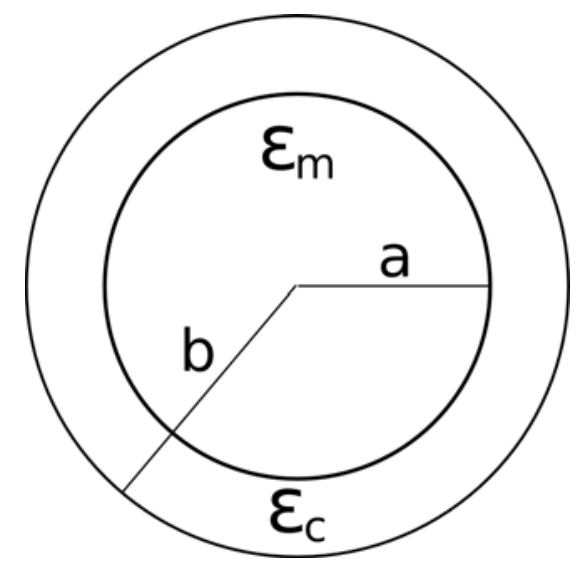

Figure 1. Geometry and relevant parameters of the coated metal cylinder.

\subsection{Decay Rates near a Coated Metallic Cylinder}

We now consider a coated metal cylinder with a core radius $a$ and a coating radius $b$. The core of this nanowire is metallic, in our case Ag, while the coating has a frequency independent dielectric constant $\varepsilon_{c}$ without any losses. Fig. 2 shows the decay rate of a quantum emitter having its transition dipole moment along the axis of the nanowire, in this case the $z$-axis, as a function of emission wavelength and of the radial position of the quantum emitter from the axis of the nanowire. We have performed the calculations for both a Ag and a Au core. 


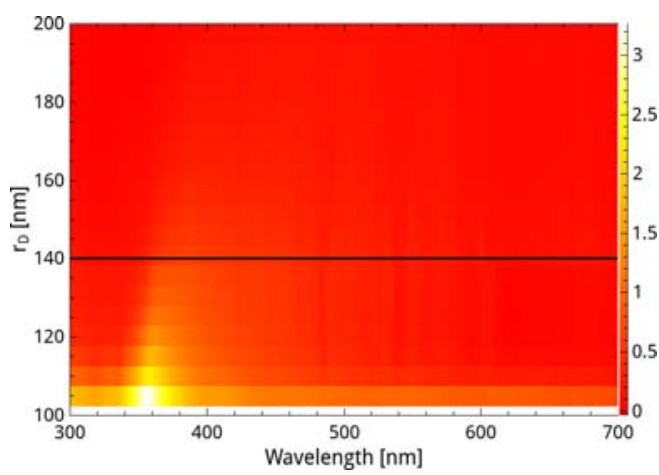

(a)

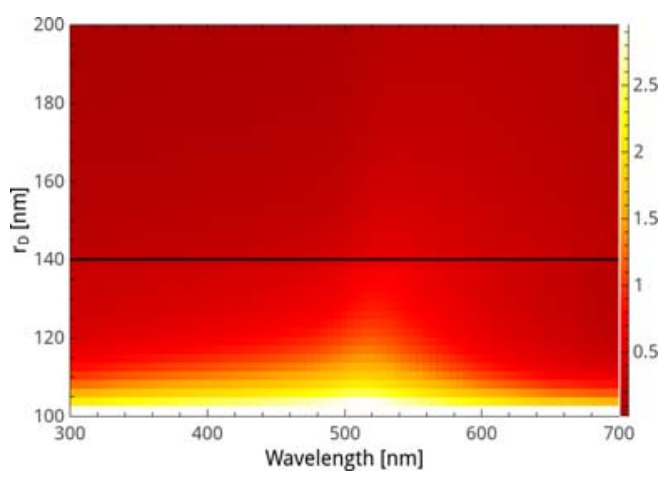

(b)

Figure 2. Wavelength and radial position dependence of the normalised decay rate of a quantum emitter in close proximity to a coated metal cylinder. (a) Ag core, $a=100 \mathrm{~nm}, b=140 \mathrm{~nm}, \varepsilon_{c}=2$; (b) Au core, $a=100 \mathrm{~nm}$, $b=140 \mathrm{~nm}, \varepsilon_{c}=2$. Logarithmic scale.

Immediately apparent is a large enhancement of the decay rate around the wavelengths (a) $\lambda=359 \mathrm{~nm}$ for the $\mathrm{Ag}$ core and (b) $\lambda=525 \mathrm{~nm}$ for the Au core. This enhancement occurs at the surface of the core, inside the coating material, and is almost non-existent outside the coating and at wavelengths off-resonance. The mechanism leading to this large enhancement of the decay rate of the emitter is the excitation of surface plasmons on the surface of the metal core by the near-field of the quantum emitter. The behaviour of the enhancement has all the hallmarks of a surface plasmon: its wavelength is resonant with the surface plasmon wavelength at the surface of $\mathrm{Ag}$ or $\mathrm{Au}$ cylinders, and it rapidly becomes negligible as the quantum emitter is moved away from the surface of the core. Furthermore, the enhancement is much sharper in wavelength for the Ag core cylinder than for the Au core one, which is again a feature of the surface plasmons on the two different metals.

By changing the thickness of the coating and its refractive index, one can control both the wavelength of the surface plasmon and thus of the largest enhancement of the decay rate as well as its confinement to the surface of the core, and thus the extent over which it can influence the lifetime of a quantum emitter.

\subsection{Energy Transfer Rates near a Coated Metallic Cylinder}

We now turn to calculation of the rate of energy transfer between a donor-acceptor pair in the presence of the coated metal cylinder, this time with a Au core. Fig. 3 shows the energy transfer rate in a plane perpendicular to the cylinder's axis, and for a donor emission wavelength of $\lambda=517 \mathrm{~nm}$ for two cylinder geometries. Both the donor and the acceptor have their transition dipole moments along the axis of the cylinder. The parameters for the two panels are given in the caption of Fig. 3.

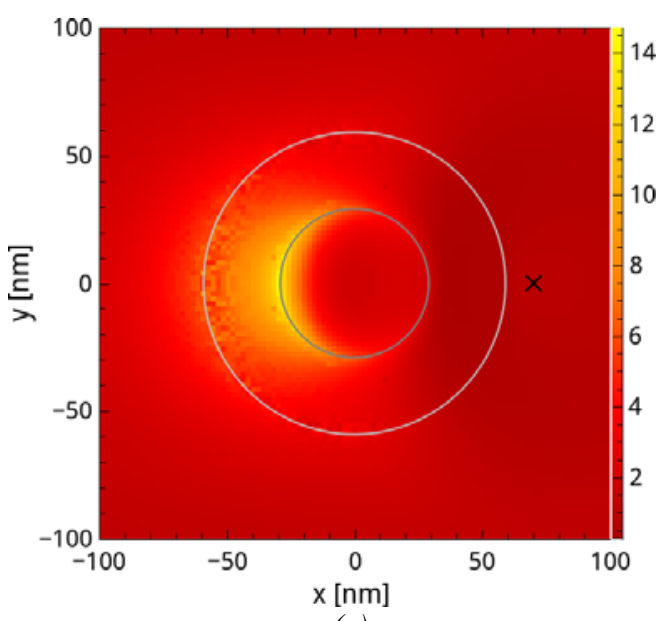

(a)

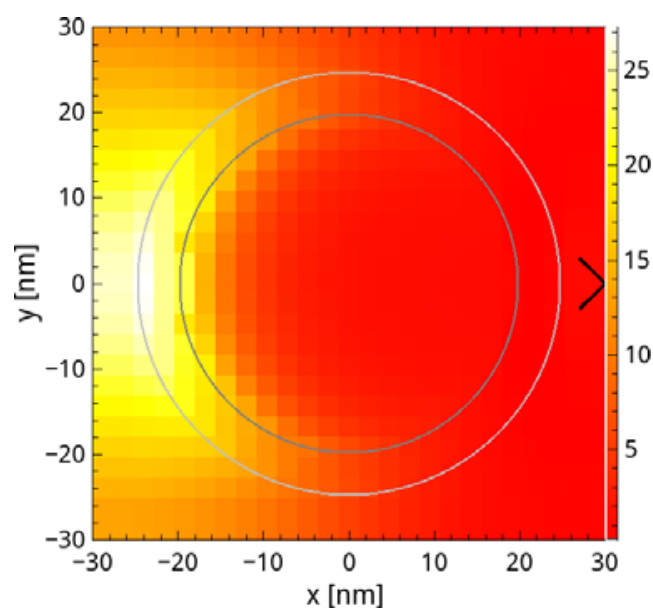

(b)

Figure 3. Normalised energy transfer rate near coated Au cylinders. The position of the donor, $r_{D}$, is indicated with a black X mark; the coated cylinder is also indicated, with $\varepsilon_{c}=2$. (a) $a=30 \mathrm{~nm}, b=60 \mathrm{~nm}, r_{D}=70 \mathrm{~nm}$; (b) $a=20 \mathrm{~nm}, b=25 \mathrm{~nm}, r_{D}=30 \mathrm{~nm}$.

From this figure one can clearly see the effect of the surface plasmons on the energy transfer rate between a donor-acceptor pair. We have chosen the radius of the coated cylinder such that scattering is minimized and the well-known focusing effect of cylinders of larger radius does not play a role. The enhancement of the energy 
transfer rate is, therefore, due entirely to the surface plasmons excited at the surface of the Au core. An interesting difference between the two panels in Fig. 3 is the fact that the largest enhancement of the energy transfer rate occurs at the surface of the Au core when the thickness of the coating is comparable to the radius of the core - panel (a) - while it occurs at the surface of the coating, when its thickness is much smaller than the radius of the coating - panel (b).

\section{SUMMARY, CONCLUSIONS AND OUTLOOK}

Having investigated the decay and energy transfer rates in the presence of a coated metal cylinder, we have shown that these rates can be considerably enhanced compared to their free-space value by the presence of the coated cylinder. We were able to attribute this effect to excitation of surface plasmons on the surface of the metal core. By changing the parameters of the coated metal cylinder, i.e. core radius, coating thickness, material of the core and coating, one can tune both the wavelength and the confinement of the surface plasmons, thus controlling the decay and energy transfer rates.

The ability to enhance and control the decay and energy transfer rates near coated metal cylinders is of particular importance in applications such as light-emitting and light-harvesting devices. By placing carefully tailored coated metal cylinder in such devices, their efficiency could be improved.

\section{ACKNOWLEDGEMENTS}

This work was supported by the Science Foundation Ireland, 10/IN.1/12975.

\section{REFERENCES}

[1] E. M. Purcell, "Spontaneous emission probabilities at radio frequencies," Physical Review, vol. 69, p. 681, 1946.

[2] K. H. Drexhage, "Influence of a dielectric interface on fluorescence decay time," Journal of Luminescence, vol. 1-2, pp. 693-701, Jan. 1970.

[3] H. Arnoldus and T. George, "Spontaneous decay and atomic fluorescence near a metal surface or an absorbing dielectric,” Physical Review A, vol. 37, no. 3, pp. 761-769, Feb. 1988.

[4] C. A. Marocico and J. Knoester, "Effect of surface-plasmon polaritons on spontaneous emission and intermolecular energy-transfer rates in multilayered geometries," Physical Review A, vol. 84, no. 5, p. 053824, Nov. 2011.

[5] X. Zhang, C. A. Marocico, M. Lunz, V. A. Gerard, Y. K. Gun'ko, V. Lesnyak, N. Gaponik, A. S. Susha, A. L. Rogach, and A. L. Bradley, "Wavelength, concentration, and distance dependence of nonradiative energy transfer to a plane of gold nanoparticles.," ACS Nano, vol. 6, no. 10, pp. 9283-90, Oct. 2012.

[6] R. Carminati, J.-J. Greffet, C. Henkel, and J. M. Vigoureux, "Radiative and non-radiative decay of a single molecule close to a metallic nanoparticle," Optics Communications, vol. 261, no. 2, pp. 368-375, May 2006.

[7] V. Klimov and M. Ducloy, "Spontaneous emission rate of an excited atom placed near a nanofiber," Physical Review A, vol. 69, no. 1, p. 013812, Jan. 2004.

[8] C. A. Marocico and J. Knoester, "Intermolecular resonance energy transfer in the presence of a dielectric cylinder," Physical Review A, vol. 79, no. 5, p. 053816, May 2009.

[9] P. Andrew and W. L. Barnes, "Energy transfer across a metal film mediated by surface plasmon polaritons," Science, vol. 306, no. 5698, pp. 1002-5, Nov. 2004.

[10] H. T. Dung, L. Knöll, and D.-G. Welsch, "Intermolecular energy transfer in the presence of dispersing and absorbing media,” Physical Review A, vol. 65, no. 4, p. 043813, Apr. 2002.

[11] M. Lunz, X. Zhang, V. A. Gerard, Y. K. Gun'ko, V. Lesnyak, N. Gaponik, A. S. Susha, A. L. Rogach, and A. L. Bradley, "Effect of Metal Nanoparticle Concentration on Localized Surface Plasmon Mediated Förster Resonant Energy Transfer,” The Journal of Physical Chemistry C, vol. 116, no. 50, pp. 26529 26534, Dec. 2012.

[12] M. Lunz, V. A. Gerard, Y. K. Gun'ko, V. Lesnyak, N. Gaponik, A. S. Susha, A. L. Rogach, and A. L. Bradley, "Surface plasmon enhanced energy transfer between donor and acceptor CdTe nanocrystal quantum dot monolayers," Nano Letters, vol. 11, no. 8, pp. 3341-5, Aug. 2011. 